\title{
AN AUDIT OF THE PHYSIOTHERAPY MANAGEMENT OF PARAPLEGIC PATIENTS WITH SACRAL PRESSURE SORES
}

\begin{abstract}
Introduction: Pressure sores are the most common complication post spinal cord injury that requires patients to be on bed rest. Patient bed rest delay rehabilitation and may lead to other complications associated with immobility. This study sought to establish the treatment interventions physiotherapists provide to patients with sacral pressure sores and the factors that they consider when deciding whether the patient should receive physiotherapy in the ward or gym.

Methods: This was a questionnaire based survey of physiotherapists working in spinal cord injury rehabilitation units in South Africa. The self designed questionnaire was sent to all the main spinal rehabilitation units in the country (14) located in Gauteng, Kwa-Zulu Natal, Western Cape, Eastern Cape and Free State provinces.

Results: Thirty-nine physiotherapists from a total of 51 completed the questionnaires (76\% response rate). The most common treatment practice for patients with sacral pressure sores was bed rest (98\%). The most common physiotherapy practices (70\%) included were upper limb muscle strengthening, upper and lower limb passive movements, positioning into prone and side lying and passive stretching. The choice of treatment environment was influenced by doctors'orders and the size, grade and duration of the pressure sores.

Conclusion: Direct involvement in pressure sore management in South Africa seem to be less than in other parts of the world. If we are to minimise the pressure sore impact, it appears like we need more focus on gait re-education and standardised ADL programmes and patient treatment in the gym to possibly maximise healing and rehabilitation.
\end{abstract}

\section{KEY WORDS: PARAPLEGIA, SACRAL PRESSURE SORES, BED REST, PRESSURE SORE TREATMENT}

\section{INTRODUCTION}

Pressure sores are the commonest complication post spinal cord injury (Aito 2003). Patients with pressures sores demonstrate significantly impaired physical and social function, self-care and mobility (Franks et al 2002). Mortality is associated with pressure sores, however, it should be noted that most often pressure sores do not cause death; rather the pressure sore is associated with a sequential decline in health status which is then associated with mortality (Allman et al

\section{Correspondence Author:}

Denisha Pather

University of the Witwatersrand

Faculty of Health Sciences

Physiotherapy Department

7 York Road, Parktown 2193

Johannesburg

Email: denishapather@hotmail.com
1995; Thomas et al 1996). Morbidities commonly associated with pressure sores include pain, depression, local infection, anaemia, osteomyelitis, and sepsis (Redelings et al 2005; Meaume et al 2005; Roth et al, 2004; Scivoletto et al 2004). The presence or development of a pressure sore can increase the length of a patient's hospital stay by an average of 10.8 days (Scott et al 2006) and the increased hospital stay is associated with higher costs and increased incidence of nosocomial infection and/ or other complications (Allman et al 1999). The average hospital treatment cost associated with stage IV pressure sores and related complications was US $\$ 129,248$ for hospital-acquired ulcers during one admission, and US\$124,327 for community-acquired ulcers over an average of four admissions among spinal cord injured patients (Brem et al 2010). The cost of treating pressure ulcers is 2.5 times the cost of preventing them (Oot-Giromini 1989). There is a dearth of information on the cost of pressure sores in South Africa.

The national pressure ulcer advisory panel in America documented the incidence of pressure sores among spinal cord injured patients to be around $62 \%$ (NPUAP 2001). The prevalence of pressure sores post spinal cord injury remains worryingly high with $27 \%$ being established by Chen et al (2005) and $38 \%$ by Ash (2002) in the United Kingdom. Thirty two percent of spinal cord injured patients were admitted to the hospital and rehabilitation setting with pre-existing pressure sores (Ash 2002).

About $46 \%$ of pressure sores are sacral sores (Ash 2002) and about two thirds of these occur in the pelvic region i.e. affecting the sacrum, coccyx, ischial tuberosities and trochanters (Garber and Rintala 2003). The high number of ischial tuberosity pressure sores among 
paraplegics is because patients with paraplegia exert $18.8 \mathrm{mmHg}$ higher interface pressure over the ischial tuberosities than unaffected people (Markhous et al 2007) due to limited postural stability.

When a patient develops pressure sores of grade II and above, especially in the sacral region, the medical prescription of choice tends to be bed rest (Rappl 2008; Virani et al 2004). The bed rest is usually for a prolonged period of time due to slow healing rates of pressure sores (Rappl 2008) and this can decrease patients' functional outcomes due to immobility associated complications.

In South Africa, epidemiological data on pressure sores is limited and no published studies from South Africa could be found that established either the direct or indirect intervention by physiotherapists in spinal cord injured patients with pressure sores. The aim of the study was thus to determine how patients with paraplegia with sacral pressure sores are being managed by physiotherapists and to establish the factors that physiotherapists take into account when deciding the treatment environment in which to manage these patients.

\section{METHOD}

\section{Study Design and Participants}

A descriptive cross sectional design using a questionnaire was used for data collection. Physiotherapists for this study were from all the 14 specialised spinal rehabilitation facilities i.e. hospitals/ clinics/practices in South Africa, which rehabilitate patients with spinal cord injuries (SCI). For inclusion in the study, physiotherapists were supposed to be involved in the treatment of patients with spinal cord injuries and were not locum or temporary employees at the rehabilitation hospitals. All physiotherapists meeting the inclusion criteria were considered for the study.

\section{Questionnaire Development}

For data collection, a self-designed questionnaire was developed. The questionnaire was developed from published clinical guidelines (literature) on the management of patients with SCI. The content validation was done using a panel of four experienced physiotherapists (greater than five years working with $\mathrm{SCI}$ ) in the field of neurology. This process also checked that the questions in the questionnaire were appropriate for the South African context. A round table discussion was held to reach agreement on which questions were to be included in the questionnaire. The questionnaire covered in part the following aspects: the demographic details of the physiotherapists and their level of experience, the use of protocols for management of patients with sacral pressure sores and establishing if the participating physiotherapists were involved in direct wound care management of sacral pressure sores. The questionnaire also sought to establish whether bed rest was often prescribed for pressure sores above grade II and the length of time this tended to be, whether the patients were receiving treatment from the therapist when in the bed or gym and the physiotherapy techniques that they used.

A pilot study was done to check the physiotherapists' understanding of the questionnaire and to iron out any unforeseen data collection difficulties. The questionnaire did not seek information on pressure sore incidence rates.

\section{Ethical Considerations}

Ethical clearance was applied for and obtained from the committee for Research on Human Subjects of the University of the Witwatersrand. Confidentiality of all information collected was ensured as the questionnaire did not require that the health professional state their name or put any identifiable data on the questionnaire.

\section{Procedure}

The head of each spinal rehabilitation facility was contacted telephonically to inform them of the study. The aims and objectives of the study were explained and any questions they may have had at that point were addressed. The email addresses of each employed physiotherapist who fitted the inclusion criteria were then obtained.

The questionnaires were then emailed to all the study participants. The questionnaires contained an information letter which described the exact details of the study and it also stated that by completing the questionnaire they were consenting to participating in the study. It requested that all responses were to be returned within two weeks. After a further three weeks a second reminder was sent out to all the participants. To ensure anonymity, email responses were sent to a different person who then printed them and gave them to the researcher.

\section{Statistical Analysis}

All data needed for the objectives were analysed using descriptive statistics and were presented either as numbers and frequencies in tables or were presented using graphs. Data were computed using International Business Machines Statistical Package for the Social Sciences (IBM SPSS) version 19.

\section{RESULTS}

\section{Demographics of the Study Sample and Response Rate}

The physiotherapists in this study were from 14 spinal rehabilitation facilities from five provinces in the country, namely Gauteng, Kwa-Zulu Natal, Western Cape, Eastern Cape and the Free State. A total of 51 (the total number of physiotherapists in the spinal rehabilitation facilities) questionnaires were sent out and 39 were received back, which amounts to a $76 \%$ response rate. Of the 39 respondents, 27(69\%) were from the private sector and the remainder from the public sector. The sample was made up of $98 \%$ females and most of the participants had $\leq 5$ years experience. Some sections of the questionnaire were not completed by some of the respondents and hence the total (n) for each section vary. Since this was a across sectional descriptive study, the available data were computed.

\section{Use of Protocols in the Rehabili- tation Centres}

Twenty physiotherapists $(51 \%)$ reported having physiotherapy treatment protocols for treatment of patients with sacral pressure sores. However, in some cases physiotherapists working in the same facilities provided different responses on the presence of treatment protocols. 


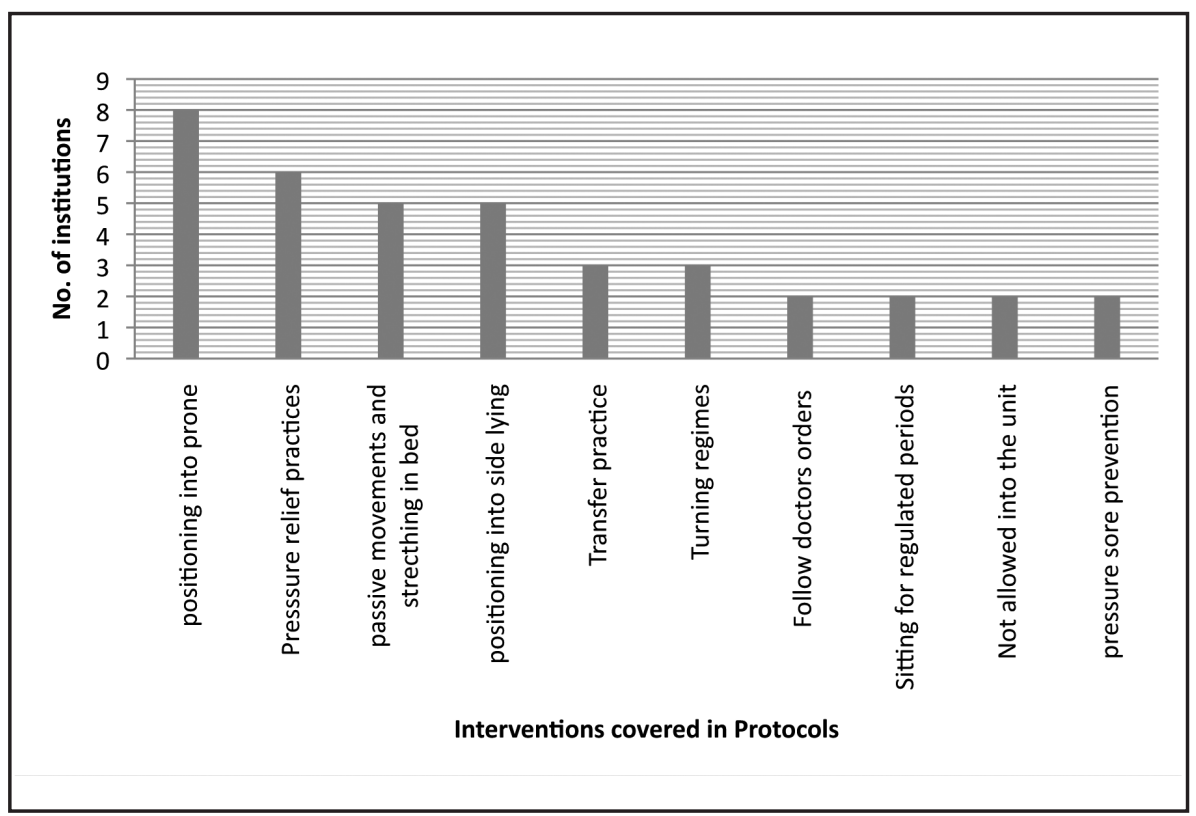

Figure 1: Interventions followed in protocols by hospital physiotherapists $(n=11)$

Table 1: Interventions provided in bed and in gym $(n=38)$

\begin{tabular}{|l|l|l|}
\hline Intervention & $\begin{array}{l}\text { In Bed } \\
\mathrm{n}(\%)\end{array}$ & $\begin{array}{l}\text { In Gym } \\
\mathrm{n}(\%)\end{array}$ \\
\hline Upper limb muscle strengthening & $36(94)$ & $38(100)$ \\
\hline Prone positioning & $35(92)$ & $34(89)$ \\
\hline Passive movements of lower limbs & $35(92)$ & $37(97)$ \\
\hline Positioning into side lying & $35(92)$ & \\
\hline Passive movements of the upper limbs & $30(78)$ & $19(50)$ \\
\hline Passive stretching of all limbs & $30(78)$ & \\
\hline Active stretching & $28(73)$ & \\
\hline Bed mobility training & $25(65)$ & $28(73)$ \\
\hline Lower limb muscle strengthening & $23(60)$ & $25(65)$ \\
\hline ADL practice in bed & $17(44)$ & $21(55)$ \\
\hline Sitting balance re-education & $8(21)$ & \\
\hline Pain treatment modalities & $8(21)$ & $9(23)$ \\
\hline Positioning into supine & $6(15)$ & \\
\hline Positioning into high sitting & $6(15)$ & \\
\hline Group therapy & $2(5)$ & $27(71)$ \\
\hline Laser treatment & $2(5)$ & $16(42)$ \\
\hline TENS treatment & $1(2)$ & $21(2)$ \\
\hline Sit to stand practice & & $25(65)$ \\
\hline Use of pack beds & & \\
\hline Use of standing frame & & \\
\hline Pressure relief exercises & & \\
\hline Mobility training & & \\
\hline Use of tilt table & & \\
\hline
\end{tabular}

The interventions covered in protocols by the various hospitals for patients with pressure sores were placed into themes and are shown in Figure $\mathbf{1 .}$ Positioning of patients in prone was the commonest $(\mathrm{n}=8)$ intervention followed with a set protocol.

From those who had protocols, 45\% said the intervention followed depended on the grade of the pressure sore while $40 \%$ said that the interventions remained the same irrespective of the grade of the pressure sore and a further $15 \%$ said that they were uncertain on whether the interventions changed with changing grades of pressure sores.

\section{Physiotherapists' Involvement in Wound Care Management}

The majority of the study sample (62\%) stated that they were not involved in direct wound care management. The distribution of the modalities used by therapists to manage pressure sores are shown in Figure 2. Ultrasound and laser were the commonest (54\%) modalities used by physiotherapists to manage pressure sores.

\section{Interventions Provided When Patients Were on Bed Rest or in the Gym.}

Ninety two percent of the physiotherapists $(n=36)$ prescribed bed rest for paraplegic patients with sacral pressure sores and 98\% $(n=38)$ treated the patients when they were on bed rest. The period of bed rest ranged from hours to months depending on the severity of the pressure sore.

The interventions provided to patients with sacral pressure sores while they were on bed rest or in the gym are shown in Table 1. Upper limb muscle strengthening was the most common intervention provided to the patients while on bed rest (94\%) and when in the gym $(70 \%)$. The majority of the therapists (98\%) stated that their patients received treatment in the gym setting as well as in the ward.

Factors Which Informed Decisions on Whether Patients Were Treated in the Gym or Ward Environment

Table 2 shows the factors that physiotherapists felt influenced their decision 
Table 2: The factors that physiotherapists felt influenced their decision on whether to treat in the ward or in the gym. $(n=39)$.

\begin{tabular}{|l|l|l|}
\hline Factor & $\mathrm{n}$ & $\%$ \\
\hline Doctors 'orders & 28 & 71 \\
\hline Grade of pressure sore & 19 & 48 \\
\hline Duration of pressure sore & 19 & 48 \\
\hline Co-morbidities present & 17 & 43 \\
\hline Size of pressure sore & 16 & 41 \\
\hline Pain levels & 12 & 30 \\
\hline Psychological status & 11 & 28 \\
\hline Urinary and bowel incontinence & 7 & 17 \\
\hline Spasticity & 6 & 15 \\
\hline Past medical history & 6 & 15 \\
\hline Infections & 3 & 7 \\
\hline Wall suction dressings or vacuum dressings & 2 & 5 \\
\hline
\end{tabular}

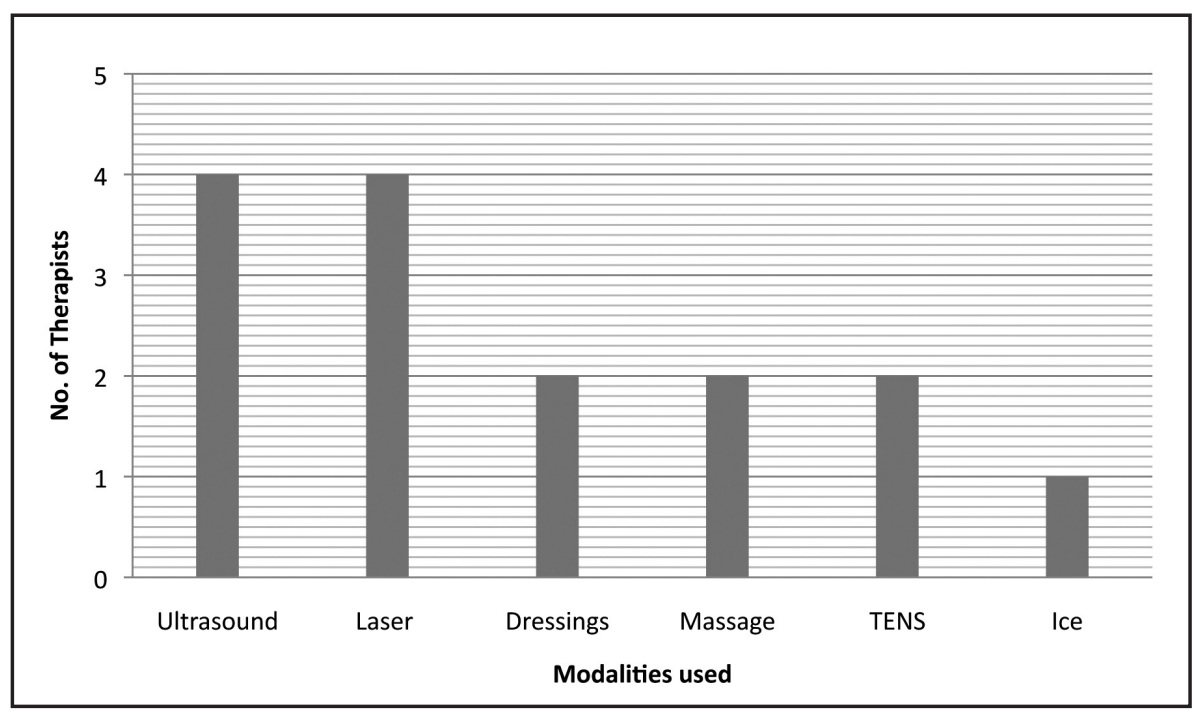

Figure 2: Direct wound care interventions $(n=15)$

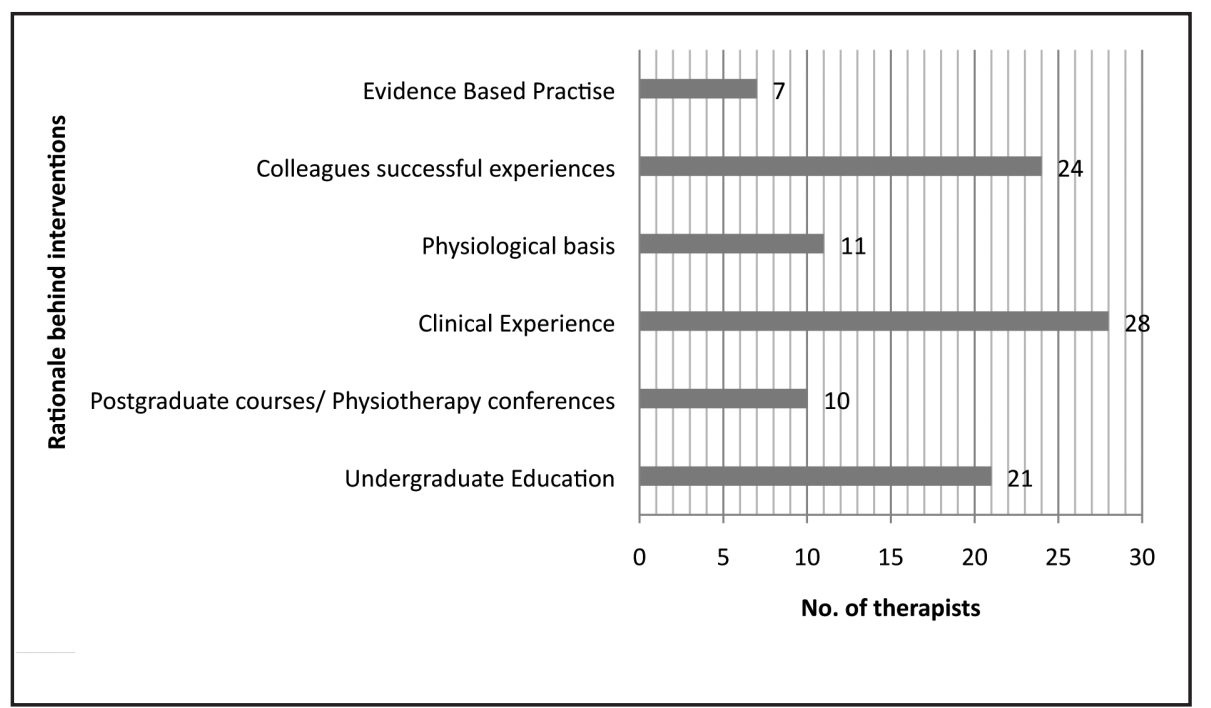

Figure 3: Background rationale physiotherapists used when choosing treatment modalities $(n=39)$ on whether to treat patients in the ward or the gym. The majority of the physiotherapists $(71 \%)$ reported that the doctors' orders influenced their decisions on whether the patient would receive treatment in the gym or the ward.

\section{Rationale Behind Physiotherapists' Interventions}

Figure 3 shows the rationale physiotherapists used when choosing physiotherapy interventions. The choice of physiotherapy modality for use when managing paraplegic patients with pressure sores was guided by among other factors their past clinical experience $(71 \%)$.

\section{Physiotherapists' Perceived Level of Knowledge of Pressure Sore Management}

Only $12(31 \%)$ of the physiotherapists felt their knowledge of management of patients with pressure sores was adequate. The reasons given for this perceived inadequacy in knowledge are shown in Figure 4. The most common reason given for the perceived inadequacy of knowledge was poor knowledge on direct wound care management.

\section{DISCUSSION}

Use of Protocols and Involvement in the Treatment of Patients with Sacral Pressure Sores

Fifty one percent of respondents stated that they had protocols they followed when treating patients. However the presence or absence of protocols varied between respondents from the same facilities which indicated either a lack of set protocols or different interpretations of the word protocol. A protocol usually consists of a set of best-practice guidelines which are to be followed for certain conditions (Field and Lohr, 1990). The respondents may have taken hospital protocol to mean the same as principles followed. Principles for the healing of pressure sores such as relieving pressure and reducing friction are followed by all therapists, however protocols in terms of: at which stage the patient is able to sit and for what time periods, the different positions the 


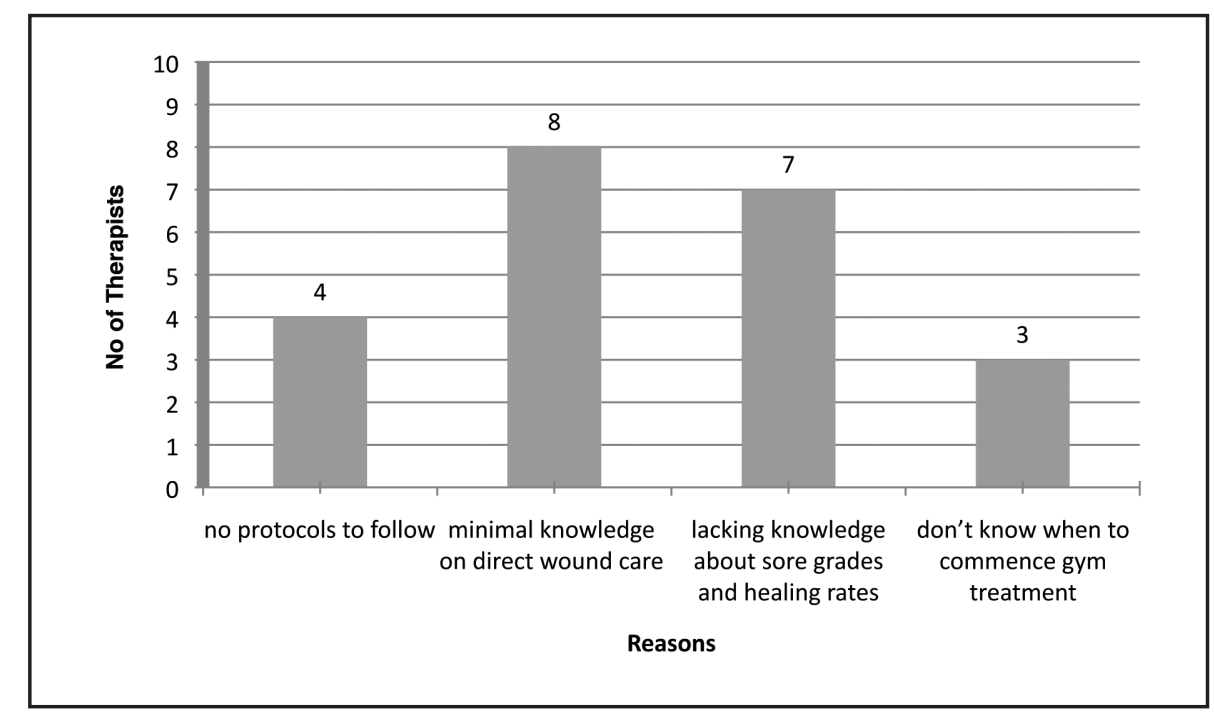

Figure 4: Reasons why physiotherapists felt their knowledge was inadequate $(n=27)$

patient can assume and direct treatment interventions do not appear to be set out in the various hospital environments. It is therefore possible that the $51 \%$ of physiotherapists that stated that they used protocols may not be an accurate percentage.

A small percentage (38\%) of the study sample reported being involved in direct wound care management. This differs to Guihan et al (2009)'s findings where they reported more than $75 \%$ of the physiotherapists to be involved in direct wound care. Eighteen percent of the respondents from this study used electrotherapy to manage pressure sores and the modalities most commonly used were ultrasound and laser. It should however be noted that the use of both laser therapy and ultrasound has not been shown to have conclusive benefits in pressure sore management which might point towards poor use of evidence by therapists when managing patients with pressure sores (Regan et al 2009; Reddy et al 2008).

\section{Physiotherapy Interventions for the Paraplegic Patient with Sacral Pressure Sores}

It was found to be common practice to place patients on bed rest if they develop sacral pressure sores. This appears to be a global practice as seen in the literature for the management of sacral pressure sores of grade II and above (Goodman et al 1999; New et al 2004; Post et al 2005). Despite patients being prescribed bed rest, the majority of the respondents $(n=38)$ stated that the patients still received treatment in both the ward and gym settings meaning patients were taken to the gym for physiotherapy when on bed rest.

Interventions that were carried out by $70 \%$ or more of the physiotherapists were taken to represent "common practice", which is fairly similar to the benchmark of $75 \%$ which was set as usual practice in Guihan et al (2009)'s study. Seventy percent of the physiotherapists indicated carrying out the following interventions when the patient was in bed: upper limb muscle strengthening, lower limb passive movements, positioning into prone and side lying as well as upper limb passive movements and passive stretching. Interventions indicated to be done in the gym setting were the same as for when on bed rest except for bed mobility training and the use of a tilt table for passive standing.

The strengthening of upper limb muscles is in keeping with general rehabilitation principles that are carried out during the rehabilitation phase of a patient with SCI (Bromley 2006; Somers 2001). However upper limb muscle strengthening in the form of functional training is needed to gain functional independence, i.e. in the form of transfers, bed mobility and gait re-education (Kloostermann et al 2009; Somers 2001). In this sample, functional upper limb strengthening does not appear to be common practice except for bed mobility practice which occurs in the gym $(n=28)$ which could affect patient ability to pressure relief to prevent pressure sores.

Standing interventions are an integral component of the rehabilitation phase post SCI to improve bone mineral density, reduce spasticity, improve digestive function and also to further prevent sacral pressure sores by removing pressure from the sacrum (Biering-Soering et al 2009; Bromley 2006; Wann-Hansson et al 2007). However standing interventions for a SCI patient with a sacral pressure sore do not appear to constitute common practice in this study sample for the patients being treated in the ward environment. However, in the gym setting, the use of the tilt table constituted common practice $(n=27)$. Other standing interventions such as the use of the standing frame $(n=21)$ and sit to stand practice $(n=16)$ were not common. In the ward setting, no standing interventions were indicated which again could possibly predispose patients to pressure sore development.

Twenty-one percent of the physiotherapists indicated sitting balance reeducation as being done in the ward and $15 \%(n=6)$ indicated the positioning of the patient into high sitting. This is not in keeping with the Agency for Health Care Policy and Research Public Service (AHCPRs) guidelines which state that a patient with a pressure sore should still be encouraged to sit once a seating assessment has been done (Bergstrom et al 1994). These low percentages can be interpreted to mean a possible high risk for pressure sore development for this cohort of patients. Rapid healing of grade III-IV pressure sores occurs when patients with sacral sores are seated correctly in positions that encourage weight shift onto the thighs and off the sacrum, i.e. upright or forward lean positions (Rosenthal 2003).

Physiotherapists in Guihan et al (2009)'s study were routinely involved in the prevention of new pressure sores by means of pressure sore education. Eight percent of this sample indicated being involved in group therapy and education, however, this aspect was not fully explored in this study and that could possibly explain the small percentage. 
Factors Taken into Consideration When Deciding the Environment in which to Manage Patients

The majority of the physiotherapists (71\%) stated that doctors' orders were a factor in deciding whether patients should be taken to the gym or not and hence what could be accomplished with the patients. This is particularly worrying in our setting where physiotherapists have first line practitioner status and does not indicate good team work where decision making should be consultative. Similar findings were established by Guihan et al (2009) where direct wound care involvement was only done on the doctor's orders, although other interventions such as sitting periods were guided by protocols. Guihan et al (2009)'s study found that the reasons for putting patients on bed rest included the presence of infection, the patient being attached to a wall suction unit or if the patient had vacuum dressings in situ. Patients with infections would need periods of isolation and a patient attached to a wall suction unit would not be able to be moved.

The participants took the grade, duration and size of the pressure sore into account before taking the patient to the gym. Pressure sores are associated with increased levels of pain and spinal rehabilitation is perceived to be painful by patients (Pellatt 2007). It is therefore possible that physiotherapists may be cautious about taking a patient who is already in pain to the gym as this may affect their adherence to therapy. In addition, the presence of pain may contribute to the development or worsening of pressure sores (Byrne et al 1996).

Spasticity interferes with functioning in patients leading to further reduction in activity (Hasima et al 2007). This decrease in the level of mobility is then a factor which contributes to the worsening or development of further pressure sores (Gelis et al 2009; Rodriquez and Garber 1994; Bryne and Salzberg 1996). This is in keeping with the caution exercised by physiotherapists when deciding whether treatment should be done in the gym or in the ward when spasticity is present. Spasticity may lead to increased levels of shear and friction which would impose limits on patient transfers in order to prevent worsening of the pressure sore.

Eighteen percent of the physiotherapists indicated that bowel and bladder continence of a patient was considered in management. Sacral pressure sores are more difficult to prevent or manage in patients with incontinence because the skin becomes over hydrated and more susceptible to shearing and friction forces (Beldon 2008). Uncontrolled urine or faecal incontinence affect treatment environment options as they are recognised risk factors for the development of pressure sores (Gelis et al 2009; Rodriquez and Garber 1994; Byrne and Salzberg 1996; Beldon 2008).

The majority of the physiotherapists indicated that their treatment interventions for patients with sacral pressure sores were guided by past clinical experiences and the successful experiences of their colleagues. Only $18 \%(n=7)$ of the physiotherapists reported using evidence based approaches to their treatment. This is worrying especially given the current emphasis on the need to base all our physiotherapy treatment modalities on evidence.

\section{Limitations of the study}

The study was purely descriptive and only sought to establish the treatment interventions physiotherapists provide to patients with sacral pressure sores and the factors that they consider when deciding whether the patient should receive physiotherapy in the ward or the gym. Consequently it does not compare the use of protocols and interventions between the private and state funded hospitals. The disparities in funding between these two institutions could possibly impact on the outcomes of the study objectives. It would also have added more depth to the study if the factors associated with the interventions could be established using regression analysis, this was however not possible given this study design.

\section{CONCLUSION AND RECOMMEN- DATIONS}

Improved education regarding the benefits and applications of direct wound care modalities need to be given to physiotherapists either during undergraduate education or with postgraduate courses. Direct involvement in pressure sore management in South Africa seem to be less than in other parts of the world. There is need to encourage more gait re-education and standardised ADL programmes to possibly maximise healing and rehabilitation as well as to encourage treatment in the gym environment as often as possible as opposed to being treated in the ward to minimise pressure sore impact. The rehabilitation team should work together to determine a goal for these patients with a programme that eliminates pressure but at the same time does not impede functional improvements.

\section{REFERENCES}

Allman RM, Goode PS, Patrick MM, Burst N, Bartolucci AA 1995 Pressure ulcer risk factors among hospitalized patients with activity limitations. JAMA. 273:865-70.

Aito S 2003 Complications during the acute phase of traumatic spinal cord lesions. Spinal Cord. 1: 629-635

Ash David 2002 An exploration of the occurrence of pressure ulcers in a British spinal injuries unit. Journal of Clinical Nursing. 11: 470-478

Beldon P 2008 Problems encountered managing pressure ulceration of the sacrum. British Journal of Community Nursing. 13 (12): 6-12

Bergstrom N, Allman R, Alvarez O, Bennett A, Carlson C 1994 The Treatment of Pressure Ulcers Guideline Panel: Pressure Ulcers Treatment Clinical Practice Guideline. Agency for Health Care Policy and Research, Public Health Service. US Department of Health and Human Services. AHCPR Publication No. 95-0652

Biering-Soering F, Hansen B, Lee B.S.B 2009 Non-pharmacological treatment and prevention of bone loss after spinal cord injury: a systematic review. Spinal Cord. 47: 508-518

Brem H, Maggi J, Nierman D, Rolnitzky L, Bell D, Renn R 2010 High Cost of Stage IV Pressure Ulcers Am J Surg. 200(4): 473-477.

Bromley I 2006 Tetraplegia and Paraplegia.A guide for physiotherapists. 6th Edition. Churchill Livingstone

Byrne DW, Salzberg CA 1996 Major risk factors for pressure ulcers in the spinal cord disabled: a literature review. Spinal Cord. 34: 255-263 
Chen Y, DeVivo MJ, Jackson AB 2005 Pressure ulcer prevalence in people with spinal cord injury: Age-period duration effects. Arch Phys Med Rehabil. 86: 1209 - 1213

Field MJ, Lohr KN (Eds). Clinical Practice Guidelines: Directions for a New Program, Institute of Medicine, Washington, DC: National Academy Press, 1990.

Franks PJ, Winterberg H, Moffatt CJ 2002 Healthrelated quality of life and pressure ulceration assessment in patients treated in the community. Wound Repair Regen.10(3):133-140.

Garber L, Rintala H 2003 Pressure ulcers in veterans with spinal cord injury: A retrospective study. Journal of rehabilitation Research and Development. 40 (5): 433- 442

Gelis A, Dupeyron A, Legros P, Benaim C, Fattal C 2009 Pressure ulcer risk factors in persons with spinal cord injury Part 2: The chronic stage. Spinal Cord. 47: 651-661.

Goodman MC, Cohen V, Armenta A, Thornby J, Netscher DT 1999 Evaluation of results and treatment variables for pressure ulcers in 48 veteran spinal cord injured patients.1Annals of Plastic Surgery Journal. 42: 665-672

Guihan M, Hastings J, Garber S.L 2009 Therapists' role in pressure ulcer management in persons with spinal cord injury. The Journal of Spinal Cord Medicine. 32: 560- 567

Hasima J.A, Bussman J.B.J, Stam H.J, Sluis T.A.R, Bergen M.P, Post M.W.M, Dallmiejer A.J, van de Woude L.H.V 2007 Physical fitness in people with spinal cord injury: The association with complications and duration of rehabilitation. Clinical Rehabilitation. 21: 932- 940

Kloosterman MGM, Snoek GJ, Jannik MJA 2009 Systematic review of the effects of exercise therapy on the upper extremity of patients with spinal cord injury.Spinal Cord. 47: 196- 203

Makhsous M , Rowles M, Ryner W, Bankard J, Nam K, Chen D, Lin F 2007 Periodically relieving ischial sitting load to decrease the risk of pressure ulcers. Archives of Physical and Medical Rehabilitation. 88: 862-870

Meaume S, Vallet D, Morere MN, Teot L 2005 Evaluation of a silver-releasing hydroalginate dressing in chronic wounds with signs of local infection. J Wound Care. 14(9):411-419.

New P, Rawicki B, Bailey M 2004 Non-traumatic spinal cord injury rehabilitation: Pressure ulcer patterns, prediction and impact. Archives of Physical Medicine and Rehabilitation. 85: 87- 93

National Pressure Ulcer Advisory Panel [NPUAP] 1989 Pressure ulcers stages. Revised 2007. Accessed 13 January 2012.

Oot-Giromini B, Bidwell FC, Heller NB Parks ML, Prebish EM, Wicks P, Williams PM1989 Pressure ulcer prevention versus treatment, comparative product cost study. Decubitus. 2(3):52-4.

Pellatt G 2007 Patients, doctors, and therapists' perceptions of professional roles in spinal cord injury rehabilitation: Do they agree? Journal of Interprofessional Care. 21:165-177

Post M, Dallmeijer A, Angenot E, van Asbeck F, van de Woude L 2005 Duration and functional outcome of spinal cord injury rehabilitation in the Netherlands. Journal of Rehabilitation Research and Development. 42: 75-86

Rappl ML 2008 Physiological changes in tissues denervated by spinal cord injury tissues possible effects on wound healing. International Wound Journal. 5: 435-444

Reddy M, Gill S, Kalkar S, Wu W, Anderson P, Rochcon P 2008 Treatment of Pressure Ulcers: A Systematic Review. Journal of American Medical Association. 300 (22): 2642- 2662

Regan MA, Teasell WR, Wolfe DL, Keast D, Mortenson W, Aubut LJ 2009 A systematic review of therapeutic interventions for pressure ulcers after spinal cord injury. Archives of Physical Medicine and Rehabilitation. 90: 213- 231.
Rosenthal MJ, Felton MR, Nastasi AE, Naliboff BD, Harker J, Navach J 2003 Healing of Advanced Pressure Ulcers by a Generic Total Contact Seat: 2 Randomized Comparisons with Low Air Loss Bed Treatments. Archives of Physical Medicine and Rehabilitation. 84:1733-1742

Redelings MD, Lee NE, Sorvillo F 2005 Pressure ulcers: more lethal than we thought? Adv Skin Wound Care. 18(7):367-372.

Rodriquez G, Garber L 1994 Prospective study of pressure ulcer risk in spinal cord injury patients. Paraplegia. 32: 150-158

Roth RS, Lowery JC, Hamill JB 2004 Assessing persistent pain and its relation to affective distress, depressive symptoms, and pain catastrophizing in patients with chronic wounds: a pilot study. Am J Phys Med Rehabil. 83(11):827-834.

Scivoletto G, Fuoco U, Morganti B, Cosentino E, Molinari M 2004 Pressure sores and blood and serum dysmetabolism in spinal cord injury patients. Spinal Cord. 42(8):473-476.

Scott JR, Gibran NS, Engrav LH, Mack CD, Rivara FP 2006 Incidence and characteristics of hospitalized patients with pressure ulcers: State of Washington, 1987 to 2000. Plast Reconstr Surg. 117(2):630-634.

Somers MF 2001 Spinal cord injury functional rehabilitation. 2nd edition. New Jersey: Prentice Hall.

Virani T, McConnell H, Tait A, Mayo S, Chee J, Cleary M, Scott C, Burris J, Chiu E 2004 Assessment and management of stage I to IV pressure ulcers. Registered Nurses Association of Ontario. Available at http://www.rnao.org/ bestpractices/ [accessed June 2010]

Wann-Hansson C, Hagell P, William A 2007 Risk factors and prevention among patients with hospital-acquired and pre-existing pressure ulcers in an acute care hospital. Journal of Clinical Nursing. 19: 1718-1727 